Integrable matrix equations related to pairs of compatible associative algebras

Odesskii, A. V. and Sokolov, V. V.

2006

MIMS EPrint: 2007.34

Manchester Institute for Mathematical Sciences

School of Mathematics

The University of Manchester

\footnotetext{
Reports available from: http://eprints.maths.manchester.ac.uk/

And by contacting: The MIMS Secretary

School of Mathematics

The University of Manchester

Manchester, M13 9PL, UK
} 


\title{
Integrable matrix equations related to pairs of compatible associative algebras
}

\author{
A V Odesskii ${ }^{1,2}$ and V V Sokolov ${ }^{1}$ \\ ${ }^{1}$ Landau Institute for Theoretical Physics, Kosygina 2, 119334, Moscow, Russia \\ 2 School of Mathematics, The University of Manchester, UK \\ E-mail: odesskii@itp.ac.ru and sokolov@itp.ac.ru
}

Received 27 April 2006, in final form 17 August 2006

Published 19 September 2006

Online at stacks.iop.org/JPhysA/39/12447

\begin{abstract}
We study associative multiplications in semi-simple associative algebras over $\mathbb{C}$ compatible with the usual one. An interesting class of such multiplications is related to the affine Dynkin diagrams of $\tilde{A}_{2 k-1}, \tilde{D}_{k}, \tilde{E}_{6}, \tilde{E}_{7}$, and $\tilde{E}_{8}$-type. In this paper we investigate in detail the multiplications of the $\tilde{A}_{2 k-1}$-type and integrable matrix ODEs and PDEs generated by them.
\end{abstract}

PACS numbers: $02.30 . S v, 02.30 . I k$, 02.30.Fn

Mathematics Subject Classification: 17B80, 17B63, 32L81, 14H70

\section{Introduction}

In the papers [1-4] different applications of the notion of compatible Lie brackets to the integrability theory have been considered. Various odd examples of compatible Lie structures have been presented in $[1,2,5]$.

A pair of compatible associative multiplications is more rigid algebraic structure than a pair of compatible Lie brackets and therefore there is a chance to construct more developed theory and some series of important examples of such multiplications.

In [6] we have studied multiplications compatible with the matrix multiplication or, in other words, linear deformations of the matrix product. It turns out that these deformations of the matrix algebra are in one-to-one correspondence with representations of certain algebraic structures, which we call $M$-structures. The case of direct sum of several matrix algebras corresponds to representations of the so-called $P M$-structures (see [6]).

The main result of [6] is a description of an important class of $M$ and $P M$-structures. The classification of these structures naturally leads to the Cartan matrices of affine Dynkin diagrams of the $\tilde{A}_{2 k-1}, \tilde{D}_{k}, \tilde{E}_{6}, \tilde{E}_{7}$, and $\tilde{E}_{8}$-type.

In this paper, we investigate integrable equations related to the affine Dynkin diagrams of $\tilde{A}$-type in details. By integrability we mean that these equations possess usual properties of integrable systems. Namely, we present Lax representations for these equations. The Lax 
representations yield first integrals and commuting flows and can be used for constructing the general solution. Moreover, our equations are bi-Hamiltonian models [16], but we do not discuss it in this paper (see conclusion). Recall some results of the papers $[1,4,6]$ adopted for our goals. For simplicity we formulate these results for the matrix algebra $\mathrm{Mat}_{n}$ but all of them remain to be true for any semi-simple associative algebra.

A multiplication $\circ$ defined on the vector space $M a t_{n}$ of all $n \times n$ matrices is said to be compatible with the matrix multiplication if the product

$$
X \bullet Y=X Y+\lambda X \circ Y
$$

is associative for any constant $\lambda$.

Since the matrix algebra is rigid, the multiplication (1.1) is isomorphic to the matrix multiplication for almost all values of the parameter $\lambda$. This means that there exists a formal series of the form

$$
S_{\lambda}=\mathbf{1}+R \lambda+O\left(\lambda^{2}\right)
$$

with the coefficients being linear operators on $M a t_{n}$, such that

$$
S_{\lambda}(X) S_{\lambda}(Y)=S_{\lambda}(X Y+\lambda X \circ Y)
$$

It follows from this formula that the multiplication $\circ$ is given by

$$
X \circ Y=R(X) Y+X R(Y)-R(X Y) .
$$

The series $S_{\lambda}$ is defined up to a transformation $S_{\lambda} \rightarrow T_{\lambda} S_{\lambda} T_{\lambda}^{-1}$, where $T_{\lambda}$ is an arbitrary element of $M a t_{n}$ of the form $T_{\lambda}=\mathbf{1}+t \lambda+O\left(\lambda^{2}\right)$. This leads to the transformation

$$
R \rightarrow R+a d_{t},
$$

which does not change the multiplication (1.4).

In the matrix case it is convenient to write down the operator $R$ in the form

$$
R(x)=a_{1} x b^{1}+\cdots+a_{p} x b^{p}+c x, \quad a_{i}, b^{i}, c \in M a t_{n}
$$

with $p$ being smallest possible.

A general construction from [4] establishes a relationship between pairs of compatible associative multiplications and integrable top-like systems. Let $\circ$ be a multiplication (1.4) compatible with the matrix product. Consider the following matrix differential equation:

$$
\frac{\mathrm{d} x}{\mathrm{~d} t}=\left[R(x)+R^{*}(x), x\right],
$$

where $*$ stands for the adjoint operator with respect to the bi-linear form trace $(x y)$. This means that, if $Q$ is a linear operator in $M_{a t}$, then $Q^{*}$ is defined by the equation trace $(Q(x) y)=\operatorname{trace}\left(x Q^{*}(y)\right)$ for all $x, y \in M a t_{n}$. In particular,

$$
R^{*}(x)=b^{1} x a_{1}+\cdots+b^{p} x a_{p}+x c .
$$

Equation (1.7) is a particular case of equation (6.9) from the paper [4] (set $u=v=$ $0, w=x$ in (6.9)). According to the results of [4] (section 6.1), equation (1.7) possesses the Lax representation

$$
\frac{\mathrm{d} L}{\mathrm{~d} t}=[A, L],
$$

where

$$
L=\left(S_{\lambda}^{-1}\right)^{*}(x), \quad A=\frac{1}{\lambda} S_{\lambda}(x) .
$$


To make these formulae constructive, we should find $S_{\lambda}$ and $S_{\lambda}^{-1}$ in a closed form, i.e. as analytic operator-valued functions. As usual, the integrals of motion for (1.7) are given by coefficients of different powers of $\lambda$ in $\operatorname{trace}\left(L^{k}\right), k=1,2 \ldots$

The simplest example of a multiplication compatible with the matrix product corresponds to the Dynkin diagram $\tilde{A}_{1}$. This multiplication $\circ$ is given by $x \circ y=x c y$, where $c$ is an arbitrary matrix. In this case we have

$$
R(x)=c x, \quad S_{\lambda}(x)=(1+\lambda c) x, \quad L=x(1+\lambda c)^{-1} .
$$

The Lax equation is equivalent to the well-known integrable matrix equation

$$
\frac{\mathrm{d} x}{\mathrm{~d} t}=x^{2} c-c x^{2} \text {. }
$$

The simplest integrals for (1.9) are given by $H_{k, 0}=\operatorname{trace}\left(x^{k}\right)$. Moreover, the $L$-operator produces an infinite set of homogeneous integrals $H_{i, j}$, where $i$ and $j$ are degrees with respect to $x$ and $c$. For example,

$$
H_{1,1}=\operatorname{trace}(x c), \quad H_{2,1}=\operatorname{trace}\left(x^{2} c\right), \quad H_{2,2}=\operatorname{trace}\left(2 c^{2} x^{2}+c x c x\right) \text {. }
$$

Equation (1.9) is Hamiltonian one with respect to the standard matrix linear Poisson bracket given by the Hamiltonian operator $a d_{x}$ and Hamiltonian function $H_{2,1}$. Indeed, (1.9) can be rewritten in the form (1.7) as $x_{t}=[x, x c+c x]$.

Matrix equations of arbitrary size like (1.9) are important because of possibility of making different reductions. For the most trivial reduction one may regard $x$ as a block matrix. In this case (1.9) becomes a system of several matrix equations for the block entries of $x$. Under reduction $x^{T}=-x, c^{T}=c(1.9)$ is a commuting flow for the $n$-dimensional Euler equation [7-9]. Another reduction of (1.9) was mentioned in [9]. Let $x$ and $c$ in (1.9) be represented by matrices of the form

$$
x=\left(\begin{array}{cccccc}
0 & u_{1} & 0 & 0 & . & 0 \\
0 & 0 & u_{2} & 0 & . & 0 \\
. & . & . & . & . & . \\
0 & 0 & 0 & 0 & . & u_{N-1} \\
u_{N} & 0 & 0 & 0 & . & 0
\end{array}\right), \quad c=\left(\begin{array}{cccccc}
0 & 0 & 0 & . & 0 & J_{N} \\
J_{1} & 0 & 0 & . & 0 & 0 \\
0 & J_{2} & 0 & . & 0 & 0 \\
. & . & . & . & . & . \\
0 & 0 & 0 & . & J_{N-1} & 0
\end{array}\right),
$$

where $u_{k}$ and $J_{k}$ are block matrices (of any dimension). It follows from equation (1.9) that $u_{k}$ satisfy the non-Abelian Volterra equation

$$
\frac{\mathrm{d}}{\mathrm{d} t} u_{k}=u_{k} \circ u_{k+1} \circ J_{k+1}-J_{k-1} \circ u_{k-1} \circ u_{k}, \quad k \in \mathbb{Z}_{N} .
$$

Integrable equation (1.9) contains one arbitrary constant matrix $c$. Such equations have been systematically investigated in [9]. In sections 1, 2 we present a series of integrable matrix equations, which depend on two constant matrices related by certain algebraic relations providing the integrability. In section 3 these results are generalized to the case of several matrix unknowns. Skew-symmetric reductions as well as reductions of (1.11)-type are available for our matrix ODEs.

Different applications of compatible associative multiplications are related to integrable deformations of the principle $G L(n)$-chiral model (see [1]). Let $\circ$ be associative multiplication compatible with the $M a t_{n} \oplus M a t_{n}$ product, $S_{\lambda}$ be the series (1.2) with the property (1.3). Define operators $T_{1}, T_{2}$ on $\mathrm{Mat}_{n}$ by means of the following decompositions:

$$
S_{\lambda}(u, 0)=(u, 0)+\lambda\left(\cdot, T_{1}(u)\right)+O\left(\lambda^{2}\right), \quad S_{\lambda}(0, v)=(0, v)+\lambda\left(T_{2}(v), \cdot\right)+O\left(\lambda^{2}\right) .
$$


Then the following hyperbolic system

$$
u_{t}=\left[u, T_{2}(v)\right], \quad v_{\tau}=\left[v, T_{1}(u)\right], \quad u, v \in M_{a t}
$$

possesses a zero-curvature representation $[L, M]=0$, where

$$
L=\frac{\mathrm{d}}{\mathrm{d} y}+\frac{1}{\lambda} S_{\lambda}(u, 0), \quad M=\frac{\mathrm{d}}{\mathrm{d} x}+\frac{1}{\lambda} S_{\lambda}(0, v) .
$$

If $T_{1}=T_{2}=\mathbf{1}$, then (1.13) is just the principle chiral model [10]. In section 3 we describe a class of integrable models (1.13) related to $P M$-structures of the $\tilde{A}_{2 k-1}$-type.

\section{Associative product of the $\tilde{A}_{3}$-type}

The $M$-structure related to $\tilde{A}_{3}$ (see [6] ) is defined by two arbitrary constant matrices $A$ and $B$ such that

$$
A^{2}=B^{2}=\mathbf{1}
$$

The corresponding associative multiplication is given by (1.4), where

$$
R(x)=A x B+B A x .
$$

This structure leads to the following integrable matrix equation

$$
x_{t}=[x, B x A+A x B+x B A+B A x] .
$$

The Lax representation (1.8) for (2.3) is given by the following explicit formulae for $S_{\lambda}$ and $S_{\lambda}^{-1}$ :

$$
\begin{aligned}
& S_{\lambda}(x)=\frac{1-q}{2} B x B+\frac{1+q}{2} x+\lambda(A x B+B A x), \\
& S_{\lambda}^{-1}(x)=\frac{1}{q}(\mathbf{1}+\lambda K)^{-1}\left(\frac{q-1}{2} B x B+\frac{1+q}{2} x+\lambda(A B x-A x B)\right),
\end{aligned}
$$

where $q=\sqrt{1-4 \lambda^{2}}, K=A B+B A$. Note that both $A$ and $B$ commute with $K$.

The canonical form (2.2) for the operator $R$ with respect to transformations (1.5) is homogeneous in $A$ and $B$ that gives rise to short expressions for $S_{\lambda}$ and $S_{\lambda}^{-1}$ irrationally depending on $\lambda$. A different form

$$
\bar{R}(x)=A x B+B A x+B x-x B
$$

provides the simplest form of the operator (1.2) with the property (1.3):

$$
\bar{S}_{\lambda}(x)=x+\lambda \bar{R}(x)
$$

but the expression for $\bar{S}_{\lambda}^{-1}$ is more complicated:

$$
\begin{gathered}
\bar{S}_{\lambda}^{-1}(x)=\frac{1}{4 \lambda^{2}-1}(\mathbf{1}+\lambda K)^{-1}\left(\lambda(A x B+B x+B A x)+2 \lambda^{2}(B x B+B A x B+A x)\right. \\
\left.-\left(\lambda+2 \lambda^{2} K\right) x B-\left(1+\lambda K-2 \lambda^{2}\right) x\right) .
\end{gathered}
$$

The corresponding Lax operators (1.8) are rational in $\lambda$.

The simplest linear and quadratic first integrals for (2.3) generated by the $L$-operator are given by

$$
\begin{aligned}
& H_{1,1}=\operatorname{trace}[x(A B+B A)], \quad H_{1,2}=\operatorname{trace}[x(A B A B+B A B A)], \\
& H_{2,1}=\operatorname{trace}\left[B A x^{2}+A x B x\right],
\end{aligned}
$$

and

$H_{2,2}=\operatorname{trace}\left[2 B A B A x^{2}+2 A B A x B x+2 B A B x A x+A B x A B x+B A x B A x\right]$. 
Remark. Equation (2.3) is the simplest example of matrix integrable equation with constant matrices related by constraints. If we assume that $A, B, x$ are block matrices of the form

$$
A=\left(\begin{array}{cc}
\mathbf{1} & 0 \\
0 & -\mathbf{1}
\end{array}\right), \quad B=\left(\begin{array}{cc}
P & \mathbf{1}+P \\
\mathbf{1}-P & -P
\end{array}\right), \quad x=\left(\begin{array}{ll}
x_{1,1} & x_{1,2} \\
x_{2,1} & x_{2,2}
\end{array}\right),
$$

then we obtain an integrable ODE with one arbitrary constant matrix $P$ and four matrix unknowns $x_{i, j}$. Furthermore, assuming that $P$ is a scalar matrix, we obtain an integrable matrix ODE with constant coefficients.

Equation (2.3) admits the following skew-symmetric reduction:

$$
x^{T}=-x, \quad B=A^{T} .
$$

Different integrable $s o(n)$-models provided by reduction (2.6) are in one-to-one correspondence with equivalence classes with respect to the $S O(n)$ gauge action of $n \times n$ matrices $A$ such that $A^{2}=\mathbf{1}$. For the real matrix $A$, a canonical form for such equivalence class can be chosen as

$$
A=\left(\begin{array}{cc}
\mathbf{1}_{p} & T \\
0 & -\mathbf{1}_{n-p}
\end{array}\right)
$$

Here $\mathbf{1}_{s}$ stands for the unity $s \times s$ matrix and $T=\left(t_{i j}\right)$, where $t_{i j}=\delta_{i j} \alpha_{i}$. This canonical form is defined by the discrete natural parameter $p$ and continuous parameters $\alpha_{1}, \ldots, \alpha_{r}$, where $p \leqslant n / 2, r=\min (p, n-p)$. A different canonical form for $A$ will be given in the next section.

For example, in the case $n=4$ the equivalence classes with $p=2$ and $p=1$ give rise to the Steklov and the Poincare integrable models $[11,12]$, correspondingly. Thus, whereas (1.9) is a matrix version of the so(4) Schottky-Manakov top [7, 13], equation (2.3)-(2.7) with $p=[n / 2]$ and $p=1$ can be regarded as $s o(n)$ generalizations for the $s o(4)$ Steklov and Poincare models (for different generalization see [14, 15]).

\section{Associative products of the $\tilde{A}_{2 k-1}$-type. Case of one matrix variable}

Let $A, B, C \in M a t_{n}$ be matrices that satisfy the following set of relations:

$$
\begin{aligned}
& A^{k}=B^{k}=\mathbf{1}, \\
& B^{i} A^{j}=\frac{\varepsilon^{-j}-1}{\varepsilon^{-i-j}-1} A^{i+j}+\frac{\varepsilon^{i}-1}{\varepsilon^{i+j}-1} B^{i+j}, \quad i+j \neq 0 \quad \bmod k, \\
& B^{i} A^{k-i}=\mathbf{1}+\left(\varepsilon^{i}-1\right) C,
\end{aligned}
$$

where $\varepsilon=\exp (2 \pi \mathrm{i} / k)$. It follows from results of [6] that formula (1.4) with

$$
R(x)=\sum_{i=1}^{k-1} \frac{1}{\varepsilon^{i}-1} A^{k-i} x B^{i}+C x,
$$

defines the associative multiplication $\circ$ compatible with the standard matrix product in $\mathrm{Mat}_{n}$. According to [6] this multiplication corresponds to the affine Dynkin diagram of the $\tilde{A}_{2 k-1^{-}}$type.

Let $T$ be any matrix such that $T^{k}-\mathbf{1}$ is invertible and

$$
A T=\varepsilon T A \text {. }
$$

Then

$$
B=(\varepsilon T-\mathbf{1})(T-\mathbf{1})^{-1} A, \quad C=T(T-\mathbf{1})^{-1}
$$

satisfy identities (3.1)-(3.3). This is a convenient way to resolve this system of identities. 
Proposition 1. The operator $S_{\lambda}$ with the property (1.3) is defined by

$$
S_{\lambda}(x)=\sum_{i=0}^{k-1} P_{i} x B^{i}, \quad P_{i}=\frac{\lambda}{\varepsilon^{i} \mu-1}(\mu T-\mathbf{1})(T-\mathbf{1})^{-1} A^{k-i},
$$

where

$$
\mu=\left(\frac{2+(k+1) \lambda}{2-(k-1) \lambda}\right)^{1 / k}
$$

The inverse operator is given by the formula

$S_{\lambda}^{-1}(x)=\sum_{i=0}^{k-1} Q_{i} x B^{i}, \quad Q_{i}=\frac{\left(\mu^{k}-1\right)^{2}}{\lambda k^{2} \mu^{k-1}\left(\mu-\varepsilon^{i}\right)}\left(\varepsilon^{k-i} T-\mathbf{1}\right)\left(\varepsilon^{k-i} \mu T-\mathbf{1}\right)^{-1} A^{k-i}$.

The corresponding Lax operator (1.8) is given by

$$
L(x)=\sum_{i=0}^{k-1} B^{i} x Q_{i}
$$

Remark. If we take operator $R$ in the following equivalent but different form

$$
\bar{R}(x)=\sum_{i=1}^{k-1} \frac{1}{\varepsilon^{i}-1}\left(A^{k-i}-1\right) x B^{i}+\left(C+\sum_{i=1}^{k-1} \frac{1}{\varepsilon^{i}-1} B^{i}\right) x
$$

then the operator $S_{\lambda}$ has the simplest form (2.5), but the formula for $S_{\lambda}^{-1}(x)$ is more complicated.

Using proposition 1, one can calculate the integrals of motion for equation (1.7) as coefficients of trace $\left(L^{k}\right), k=1,2 \ldots$. For example, the simplest quadratic integral is given by

$$
H=\operatorname{trace}(x R(x)) \text {. }
$$

Equation (1.7) can be written in the Hamiltonian form as

$$
\frac{\mathrm{d} x}{\mathrm{~d} t}=[x, \operatorname{grad} H] .
$$

The skew-symmetric reduction is given by

$$
B=\left(A^{t}\right)^{-1}, \quad C=\frac{\varepsilon}{1-\varepsilon}\left(A^{t} A-1\right),
$$

where $A$ should satisfy the following additional constraint

$$
\left(A^{t} A-\varepsilon^{-1}\right) A\left(A^{t} A-1\right)=\varepsilon\left(A^{t} A-1\right) A\left(A^{t} A-\varepsilon^{-1}\right) .
$$

It turns out that

$$
A=\sqrt{z_{1}} e_{k, 1}+\sum_{i=2}^{k} \sqrt{z_{i}} e_{i-1, i}
$$

where $z_{j+1}=f\left(z_{j}\right)$,

$$
f(z)=\frac{1}{1+\varepsilon-\varepsilon z},
$$

defines a skew-symmetric reduction with one arbitrary parameter $z_{1}$. In particular, for $k=2$ we have

$$
A=\left(\begin{array}{cc}
0 & \alpha \\
\alpha^{-1} & 0
\end{array}\right)
$$

where $\alpha$ is an arbitrary parameter. 
Remark. Any block-diagonal matrix with blocks (of different side) of the form (3.9) also provides a non-trivial skew-symmetric reduction. If we take $p$ blocks of the size 1 and the remaining blocks of the size 2 , we get a canonical form for matrix $A$ equivalent but different from (2.7).

\section{Associative products of the $\tilde{A}_{2 k-1}$-type. Case of $m$ matrix variables}

In this section we consider associative multiplications compatible with the product in the direct sum of $m$ copies of $M a t_{n}$. We use the notation $x=\left(x_{1}, \ldots, x_{m}\right)$, where $x_{1}, \ldots, x_{m} \in M a t_{n}$, for $x \in\left(M a t_{n}\right)^{m}$. The standard multiplication in $\left(M_{a t}\right)^{m}$ is given by the formula $x y=\left(x_{1} y_{1}, \ldots, x_{m} y_{m}\right)$. Using results obtained in [6], the following statement can be proved.

Proposition 2. Let $T, B$ be matrices such that

$$
B^{k}=1, \quad B T=\varepsilon T B .
$$

Fix generic numbers $\lambda_{1}, \ldots \lambda_{m}, t_{1}, \ldots, t_{m} \in \mathbb{C}$ such that $T^{k}-\lambda_{\alpha}^{k}$ are invertible for all $\alpha=1, \ldots, m$. Then formula (1.4) with

$R(x)=\left(R_{1}(x), \ldots, R_{m}(x)\right)$,

$R_{\alpha}(x)=\sum_{(i, \beta) \neq(0, \alpha)} \frac{t_{\beta}}{\varepsilon^{i} \lambda_{\beta}-\lambda_{\alpha}} \cdot \frac{T-\lambda_{\alpha}}{\varepsilon^{-i} T-\lambda_{\beta}} B^{-i} x_{\beta} B^{i}+\left(\frac{t_{\alpha}}{T-\lambda_{\alpha}}-\sum_{(i, \beta) \neq(0, \alpha)} \frac{\varepsilon^{i} t_{\beta}}{\varepsilon^{i} \lambda_{\beta}-\lambda_{\alpha}}\right) x_{\alpha}$

defines the following associative product compatible with the standard product in $\left(\mathrm{Mat}_{n}\right)^{m}$ :

$$
x \circ y=\left((x \circ y)_{1}, \ldots,(x \circ y)_{m}\right),
$$

where

$$
\begin{aligned}
(x \circ y)_{\alpha}= & \sum_{(i, \beta) \neq(0, \alpha)} \frac{t_{\beta}}{\varepsilon^{i} \lambda_{\beta}-\lambda_{\alpha}}\left(\frac{T-\lambda_{\alpha}}{\varepsilon^{-i} T-\lambda_{\beta}} B^{-i} x_{\beta} B^{i} y_{\alpha}+x_{\alpha} \frac{T-\lambda_{\alpha}}{\varepsilon^{-i} T-\lambda_{\beta}} B^{-i} y_{\beta} B^{i}\right. \\
& \left.-\frac{T-\lambda_{\alpha}}{\varepsilon^{-i} T-\lambda_{\beta}} B^{-i} x_{\beta} y_{\beta} B^{i}\right)+t_{\alpha} x_{\alpha} \frac{1}{T-\lambda_{\alpha}} y_{\alpha}-\sum_{(i, \beta) \neq(0, \alpha)} \frac{\varepsilon^{i} t_{\beta}}{\varepsilon^{i} \lambda_{\beta}-\lambda_{\alpha}} x_{\alpha} y_{\alpha} .
\end{aligned}
$$

Remark. The formulae from section 2 after elimination of matrices $A^{i}, C$ via (3.5) coincides with the corresponding formulae from proposition 2 with $m=1$.

Proposition 3. For the multiplication from proposition 2 the operator (1.2) with the property (1.3) is given by

$$
S_{\lambda}(x)=\left(S_{1}(x), \ldots, S_{m}(x)\right),
$$

where

$S_{\alpha}(x)=\sum_{i, \beta} \frac{\lambda t_{\beta}\left(T-\mu_{\alpha}\right)}{\left(\varepsilon^{i} \lambda_{\beta}-\mu_{\alpha}\right)\left(\varepsilon^{-i} T-\lambda_{\beta}\right)} B^{-i} x_{\beta} B^{i}$.

The Lax operator (1.8) is defined by the formulae

$$
L(x)=\left(L_{1}(x), \ldots, L_{m}(x)\right),
$$

where

$$
\begin{aligned}
L_{\alpha}(x)=-\sum_{i, \beta} & \frac{\prod_{s}\left(\lambda_{\alpha}^{k}-\mu_{s}^{k}\right)\left(\lambda_{s}^{k}-\mu_{\beta}^{k}\right)}{k^{2} \lambda_{\alpha}^{k-1} \mu_{\beta}^{k-1}\left(\lambda_{\alpha}-\varepsilon^{i} \mu_{\beta}\right) \prod_{s \neq \alpha}\left(\lambda_{\alpha}^{k}-\lambda_{s}^{k}\right) \prod_{s \neq \beta}\left(\mu_{\beta}^{k}-\mu_{s}^{k}\right)} \\
& \times B^{i} x_{\beta} \frac{T-\lambda_{\alpha}}{t_{\alpha} \lambda\left(\varepsilon^{-i} T-\mu_{\beta}\right)} B^{-i} .
\end{aligned}
$$


Here $\mu_{\alpha}=\mu_{\alpha}(\lambda)$ are algebraic functions in $\lambda$ such that $\mu_{\alpha}(0)=\lambda_{\alpha}$ and

$$
\sum_{\gamma} \frac{t_{\gamma} \lambda_{\gamma}^{k-1}}{\lambda_{\gamma}^{k}-\mu_{\alpha}^{k}}=\frac{1}{k \lambda}, \quad \alpha=1, \ldots, m .
$$

Remark. In some sense, the Lax operator constructed in proposition 3 is a multi-pole analogue of operator (3.8).

To find the explicit form of integrable equations related to this Lax operator, one needs several first coefficients of the Taylor expansions of $S_{\lambda}$ and $L$. We have

$$
\begin{gathered}
\mu_{\alpha}=\lambda_{\alpha}-t_{\alpha} \lambda-t_{\alpha}\left(\frac{k-1}{2} \cdot \frac{t_{\alpha}}{\lambda_{\alpha}}+k \sum_{\gamma \neq \alpha} \frac{t_{\gamma} \lambda_{\gamma}^{k-1}}{\lambda_{\gamma}^{k}-\lambda_{\alpha}^{k}}\right) \lambda^{2}+O\left(\lambda^{3}\right), \\
S_{\alpha}(x)=x_{\alpha}+\left(\frac{t_{\alpha}}{T-\lambda_{\alpha}}-\frac{k-1}{2} \cdot \frac{t_{\alpha}}{\lambda_{\alpha}}-k \sum_{\gamma \neq \alpha} \frac{t_{\gamma} \lambda_{\gamma}^{k-1}}{\lambda_{\gamma}^{k}-\lambda_{\alpha}^{k}}\right) x_{\alpha} \lambda \\
+\sum_{(i, \beta) \neq(0, \alpha)} \frac{t_{\beta}\left(T-\lambda_{\alpha}\right)}{\left(\varepsilon^{i} \lambda_{\beta}-\lambda_{\alpha}\right)\left(\varepsilon^{-i} T-\lambda_{\beta}\right)} B^{-i} x_{\beta} B^{i} \lambda+O\left(\lambda^{2}\right), \\
L_{\alpha}(x)=x_{\alpha}-x_{\alpha}\left(\frac{t_{\alpha}}{T-\lambda_{\alpha}}-\frac{k-1}{2} \cdot \frac{t_{\alpha}}{\lambda_{\alpha}}-k \sum_{\gamma \neq \alpha} \frac{t_{\gamma} \lambda_{\gamma}^{k-1}}{\lambda_{\gamma}^{k}-\lambda_{\alpha}^{k}}\right) \lambda \\
-\sum_{(i, \beta) \neq(0, \alpha)} B^{i} x_{\beta} \frac{t_{\beta}\left(T-\lambda_{\alpha}\right)}{\left(\varepsilon^{i} \lambda_{\beta}-\lambda_{\alpha}\right)\left(\varepsilon^{-i} T-\lambda_{\beta}\right)} B^{-i} \lambda+O\left(\lambda^{2}\right) .
\end{gathered}
$$

The integrable system (1.7) has the form

$$
\begin{gathered}
\frac{\mathrm{d} x_{\alpha}}{\mathrm{d} t}=\left[\frac{t_{\alpha}}{T-\lambda_{\alpha}} x_{\alpha}+x_{\alpha} \frac{t_{\alpha}}{T-\lambda_{\alpha}}+\sum_{(i, \beta) \neq(0, \alpha)}\left(\frac{t_{\beta}\left(T-\lambda_{\alpha}\right)}{\left(\varepsilon^{i} \lambda_{\beta}-\lambda_{\alpha}\right)\left(\varepsilon^{-i} T-\lambda_{\beta}\right)} B^{-i} x_{\beta} B^{i}\right.\right. \\
\left.\left.+B^{i} x_{\beta} \frac{t_{\beta}\left(T-\lambda_{\alpha}\right)}{\left(\varepsilon^{i} \lambda_{\beta}-\lambda_{\alpha}\right)\left(\varepsilon^{-i} T-\lambda_{\beta}\right)} B^{-i}\right), x_{\alpha}\right] .
\end{gathered}
$$

It can be written in the Hamiltonian form as

$$
\frac{\mathrm{d} x}{\mathrm{~d} t}=[x, \operatorname{grad} H],
$$

where $H=\sum_{\alpha} \operatorname{trace}\left(x_{\alpha} R_{\alpha}(x)\right)$.

Remark. All these constructions including the integrable system are valid for the case $k=1$ also. In this case $B=\mathbf{1}, \varepsilon=1$ and $T$ is an arbitrary matrix. If also $m=1$, then the system is equivalent to (1.9).

Another application of propositions 2, 3 in the case $m=2$ is the integrable PDE (1.13). To write down this system explicitly, we need to find the operators $T_{1}$ and $T_{2}$ defined by (1.12). The asymptotic formula for $S_{\lambda}$ given above yields

$$
T_{1}(x)=\sum_{i} \frac{t_{1}\left(T-\lambda_{2}\right)}{\left(\varepsilon^{i} \lambda_{1}-\lambda_{2}\right)\left(\varepsilon^{-i} T-\lambda_{1}\right)} B^{-i} x B^{i},
$$




$$
T_{2}(x)=\sum_{i} \frac{t_{2}\left(T-\lambda_{1}\right)}{\left(\varepsilon^{i} \lambda_{2}-\lambda_{1}\right)\left(\varepsilon^{-i} T-\lambda_{2}\right)} B^{-i} x B^{i} .
$$

\section{Conclusion}

In this paper we have described representations of $M$ and $P M$-structures related to the affine Dynkin diagrams of the $\tilde{A}_{2 k-1}$-type and have presented Lax operators for the corresponding matrix top-like systems. Actually, these systems are bi-Hamiltonian models [16]. We are planning to describe the Hamiltonian properties of these systems in a separate paper. Besides of that representations of $P M$-structures related to the affine Dynkin diagrams of the $\tilde{D}_{k}, \tilde{E}_{6}, \tilde{E}_{7}, \tilde{E}_{8}$-types and the corresponding integrable models should be described explicitly. A systematic investigation of reductions of matrix models constructed in this paper seems to be an interesting problem. In this paper we have also considered integrable PDEs of the chiral model type related to $P M$-structures with $m=2$. Compatible associative multiplications described by $M$ and $P M$-structures can be used for the construction of integrable PDEs of the Landau-Lifshitz type in accordance with results of [17].

\section{Acknowledgments}

The second author (VS) is grateful to USTC (Hefei, China) for hospitality. The research was partially supported by RFBR grants 05-01-00775, 05-01-00189 and NSh grants 1716.2003.1, 2044.2003.2.

\section{References}

[1] Golubchik I Z and Sokolov V V 2002 Compatible Lie brackets and integrable equations of the principle chiral model type Funct. Anal. Appl. 36 172-81

[2] Golubchik I Z and Sokolov V V 2005 Factorization of the loop algebras and compatible Lie brackets J. Nonlinear Math. Phys. 12 343-50

[3] Golubchik I Z and Sokolov V V 2006 Compatible Lie brackets and Yang-Baxter equation Theor. Math. Phys. 146 159-69

[4] Golubchik I Z and Sokolov V V 2004 Factorization of the loop algebra and integrable top-like systems Theor. Math. Phys. 141 3-23

[5] Bolsinov A V and Borisov A V 2002 Lax representation and compatible Poisson brackets on Lie algebras Math. Notes 72 11-34

[6] Odesskii A V and Sokolov V V 2005 Algebraic structures connected with pairs of compatible associative algebras Preprint math.QA/0512499

[7] Manakov S V 1976 Note on the integration of Euler's equations of the dynamics of an $n$-dimensional rigid body Funct. Anal. Appl. 10 93-4

[8] Fedorov Yu N 1995 Integrable systems, Lax representations, and confocal quadrics. Dynamical systems in classical mechanics (Amer. Math. Soc. Transl. Ser. 2) vol 168 (Providence, RI: American Mathematical Society) 173-99

[9] Mikhailov A V and Sokolov V V 2000 Integrable ODEs on associative algebras Comm. in Math. Phys. 211 231-51

[10] Pohlmeyer K 1976 Integrable Hamiltonian systems and interactions through quadratic constraints Commun. Math. Phys. 46 207-21

[11] Stekloff W 1909 Sur le mouvement d'un corps solide ayant une cavité de forme ellipsoidale remplie par un liquide incompressible en sur les variations des latitudes (French) Ann. Fac. Sci. Toulouse Sci. Math. Sci. Phys. (3) 1 145-256

[12] Poincare H 1910 Sur la precession des corps deformables Bull. Astron. 27 321-56

[13] Schottky F 1891 Uber das analytische Problem der Rotation eines starren Körpers in Raume von vier Dimensionen Sitz. drer Königligh preussischen Acad. Wiss. Berl. 13 227-32 
[14] Ratiu T 1982 Euler-Poisson equations on Lie algebras and the $N$-dimensional heavy rigid body $A m . J$. Math. 103 409-48

[15] Bolsinov A V and Fedorov Yu N 1992 Multi-dimensional integrable generalization of the Steklov-Lyapunov systems Vestnik MGU 6 53-6

[16] Magri F 1978 A simple model of the integrable Hamiltonian equation J. Math. Phys. $191156-62$

[17] Golubchik I Z and Sokolov V V 1999 Generalized Heizenberg equations on Z-graded Lie algebras (Russian) Teor. Mat. Fiz. 120 (no 2) 248-55

Golubchik I Z and Sokolov V V 1999 Generalized Heizenberg equations on Z-graded Lie algebras Theoret. Math. Phys. 120 (no 2) 1019-25 